\title{
A worksite health promotion intervention improved employees' nutrition
}

Biener L, Glanz K, McLerran D, et al. Impact of the Working Well Trial on the worksite smoking and nutrition environment. Health Educ Behav 1999 Aug;26:478-94.

QUESTION: Can a worksite health promotion intervention change the physical and social environment related to the dietary and smoking behaviours of employees?

\section{Design}

Randomised (allocation not concealed), unblinded, controlled trial with 3 years of follow up (The Working Well Trial).

\section{Setting}

114 worksites in the US.

\section{Participants}

20801 employees (68\% men, 92\% white) from 114 worksites. Worksites represented areas of manufacturing, communication, public service, and utilities and were matched for size, presence of a cafeteria site, type of smoking policy, company type, sex distribution, blue or white collar predominance, and baseline survey response. Randomisation occurred within matched pairs. Work site follow up was $97 \%$ and participant follow up was $88 \%$.

\section{Intervention}

57 worksites (10 071 participants) were allocated to the nutrition and smoking intervention group and 57 (10 730 participants) were allocated to the control group. Interventions aimed to achieve changes in both social norms and the physical environment. Employees at intervention worksites formed employee advisory boards, which collaborated with an interventionist from the study (eg, proposed ways of increasing accessibility to healthy foods, and developed and implemented company policies to support healthy eating and smoking cessation). Employees at control worksites received baseline survey data and used optional distribution of printed materials and self help programmes.

\section{Main outcome measures}

Changes in worksite physical environment and social norms related to nutrition and smoking were assessed by surveys of employees and key organisational informants.

\section{Main results}

Compared with employees at control worksites, those at intervention worksites perceived a change in both the physical environment (access to healthy food and nutrition information, $\mathrm{p}<0.001)$ and social environment (coworker support for low fat dietary choices and management concern about employees' nutrition, $\mathrm{p}<0.001)$ related to nutrition at the worksite. Informants at intervention worksites also perceived healthy changes in the nutrition environment (nutrition labelling, $\mathrm{p}<0.001$, and Healthy Food Environment Index score, $\mathrm{p}=0.01$ ). Generally, no differences existed between groups in employee or informant perceptions of the change in physical or social environment related to smoking; however, employees who smoked at intervention worksites reported greater encouragement to stop smoking from coworkers and employers $(\mathrm{p}<0.05)$. Both intervention and control worksites showed improvements in worksite smoking policies and social norms about smoking.

\section{Conclusion}

A worksite health promotion intervention improved the physical and social environment related to nutrition behaviours but not smoking behaviours of employees.

\section{COMMENTARY}

The methods and results of this study will be of particular interest to health professionals in public health and occupational health fields. This study by Biener $e t$ al represents the largest published, English language trial done on worksite nutrition and smoking interventions. A previous report of this study described small but significant reductions in workers' fat consumption and an increase in fruit and vegetable intake, but no effect on smoking cessation. ${ }^{1}$ The only other comparable trial was much smaller (1762 workers, 13 companies) and found a similar increase in total fruit and vegetable intake and a reduction in margarine and butter intake. ${ }^{2}$ This article reports on physical and social environmental changes that occurred at 114 worksites.

There was a broad range of participants from many worksites in the US with populations that included white collar and blue collar workers. We know that all of the participants were employed, but the authors did not examine the effects of the wage structure or social status of the participants. Furthermore, individual economic level and marital status may have affected the amount of money and time available to make appropriate nutritional choices, not only at work but also at home. Further subgroup analysis may have provided information on whether certain groups benefited more from the intervention (eg, white collar workers).

It is generally understood that smoking cessation results vary with an individual's personal desire to stop smoking. Many employers in this same time period (1985-92) used various forms of smoking bans in their workplaces as a direct result of public awareness. This may have meant that the control sites also received an intervention minimising potential group differences in outcomes.

Although the results are highly relevant to worksites, administrators of these settings might be more likely to adopt this intervention if it was accompanied by data about its impact on productivity through measures such as employee sickness and absence.

Brian Verrall, COHN, DOHS, MSc Manager, Occupational Health and Safety Faculty of Health Sciences, McMaster University Hamilton, Ontario, Canada

For correspondence: Dr L Biener, Center for Survey Research University of Massachusetts, 100 Morrissey Boulevard, Boston, MA 02125, USA Fax +16172877210.
1 Sorensen G, Thompson B, Glanz K, et al. Work site-based cancer prevention: primary results from the Working Well Trial. Am J Public Health 1996;86:939-47.

2 Hunt MK, Hebert JR, Sorensen G, et al. Impact of a worksite Journal of Nutrition Education 1993;25:236-44. cancer prevention program on eating patterns of workers. 